\title{
De novo malignant solitary fibrous tumor of the kidney
}

\author{
Tsan-Yu Hsieh ${ }^{1,4}$, Yi-Che ChangChien ${ }^{1,4}$, Wen-Hsiang Chen ${ }^{2,4}$, Siu-Chung Chen ${ }^{3,4}$, Liang-Che Chang ${ }^{1,4}$, \\ Cheng-Cheng Hwang ${ }^{1,4}$, Hui-Ping Chein ${ }^{1,4}$ and Jim-Ray Chen ${ }^{1, *^{*}}$
}

\begin{abstract}
The kidney is a relatively infrequent site for solitary fibrous tumor (SFT). Among the previously reported cases, only two cases of malignant renal SFT developing via dedifferentiation from a pre-existing benign SFT have been reported. Here we reported a case of de novo malignant renal SFT clinically diagnosed as renal cell carcinoma in a 50-year-old woman. The tumor was circumscribed but unencapsulated and showed obvious hemorrhagic necrosis. Microscopically, the tumor was composed of patternless sheets of alternating hypercellular and hypocellular areas of spindle cells displaying mild to moderate nuclear atypia, frequent mitoses up to 8 per 10 high power fields, and a 20\% Ki-67 proliferative index. Immunohistochemical studies revealed reactivity for CD34, CD99 and vimentin, with no staining for all other markers, confirming the diagnosis of SFT. No areas of dedifferentiation were seen after extensive sampling. Based on the pathologic and immunohistochemical features, a diagnosis of de novo malignant renal SFT was warranted. Our report expands the spectrum of malignant progression in renal SFTs. Even though this patient has been disease-free for 30 months, long-term follow-up is still mandatory.
\end{abstract}

Keywords: solitary fibrous tumor, kidney, malignant, de novo, dedifferentiation, CD34

\section{Backround}

Solitary fibrous tumors (SFTs) are distinctive mesenchymal tumors most commonly described as pleural-based lesions; however they can develop at any extrapleural anatomic site [1]. Although the clinical course of SFTs is rather unpredictable, the prognosis of SFTs is generally favorable. It is estimated that $10 \%$ to $15 \%$ of intrathoracic SFTs and up to $10 \%$ of extrathoracic SFTs will recur and/ or metastasize $[2,3]$, therefore $\mathrm{SFT}$ is regarded as an "intermediate malignant, rarely metastasizing" neoplasm [4]. Microscopic features associated with malignancy in both intrathoracic and extrathoracic SFTs include nuclear atypia, increased cellularity and more than 4 mitoses per 10 high power fields [4,5]. An additional factor conferring a worse prognosis in SFTs is dedifferentiation or sarcomatous overgrowth, which represents an abrupt transition to a morphologically anaplastic component [6]. The kidney is a relatively infrequent site for SFT, with at least 36 cases reported in a review article [7]. The vast majority of renal SFTs are histologically

\footnotetext{
* Correspondence: jimrchen@cgmh.org.tw

'Department of Pathology, Chang Gung Memorial Hospital, Keelung, Taiwan Full list of author information is available at the end of the article
}

benign and only two cases of malignant renal SFTs developing via dedifferentiation or sarcomatous overgrowth from a pre-existing benign SFT have been reported $[7,8]$. Here we report the first case of de novo malignant renal SFT without dedifferentiation and thus expand the spectrum of malignant progression in renal SFTs.

\section{Case presentation}

\section{Clinical summary}

A 50-year-old woman was admitted to our hospital with one-month history of soreness and pain in her right flank, without gross hematuria or other constitutional symptoms. Laboratory findings were unremarkable. Physical examination revealed a palpable right flank mass. A computed topography $(\mathrm{CT})$ of the abdomen showed a huge necrotic tumor occupying the perirenal space of right kidney without evidence of either local invasion or lymphadenopathy (Figure 1). The patient underwent right radical nephrectomy under a pre-operative diagnosis of American Joint Committee on Cancer (AJCC) stage II (T2aN0) renal cell carcinoma. Post-operation course was smooth. Neither chemotherapy nor radiation therapy was given.

\section{Biomed Central}




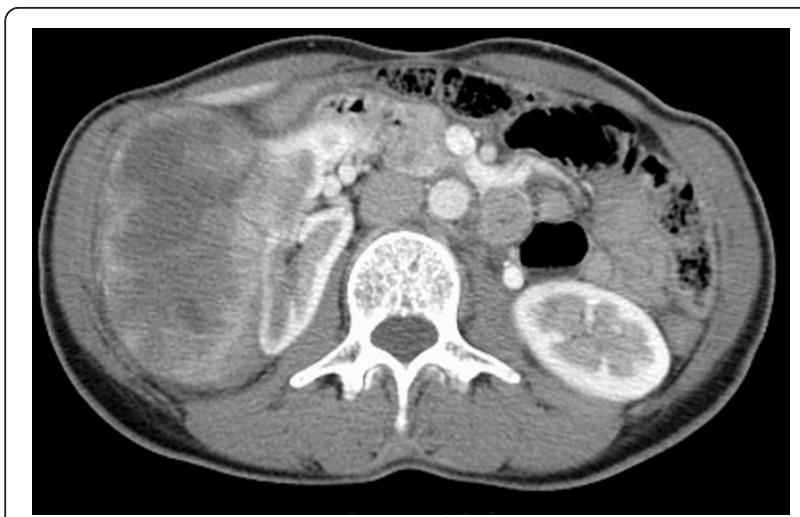

Figure 1 CT of the abdomen. Arterial phase images of dynamic computed topography scan showed a highly necrotic tumor compressing the renal parenchyma without either invasion to surrounding tissues or local lymphadenopathy.

She has been well without evidence of recurrence or metastasis for 30 months.

\section{Pathologic findings}

A nephrectomy specimen $(15 \times 9 \times 7 \mathrm{~cm}, 670 \mathrm{~g})$ with attached ureter and perirenal fibroadipose tissue was received. The specimen was bisected to reveal a $9 \times 9 \times$ $6 \mathrm{~cm}$ circumscribed but unencapsulated tumor occupying the perirenal space of the upper and middle poles of kidney. The tumor was firm and showed a yellowish white to tan-gray, myxoid and lobulated cut surface with prominent hemorrhage and necrosis (Figure 2). Microscopically, the tumor showed proliferation of spindle cells arranging in a patternless architecture (Figure
3A) with a combination of alternating hypercellular and hypocellular areas (Figure 3B). Haphazard, storiform, or short fascicular arrangements of spindle cells in a loose myxoid to fibrous stroma containing dense collagen fibers were also seen (Figure 3C). Dilated and branching hemangiopericytoma-like vessels were frequently observed (Figure 3D). Tumor cells had plump, fusiform, or elongated hyperchromatic nuclei with mild to moderate pleomorphism and indistinct cell borders and frequent mitoses up to 8 per 10 high power fields. Abnormal mitoses were occasionally seen (Figure 3E). Tumor necrosis was evidently present (Figure 3F). We did not find any areas of dedifferentiation after extensive tumor sampling.

Immunohistochemically, the tumor showed weak CD34 positivity (Figure 4A) and diffusely strong CD99 (Figure 4B) and vimentin staining. They stained negatively for bcl-2 protein, S-100 protein, NSE, muscle markers, cytokeratin, CD117(C-kit), p53 and HMB-45. Ki-67 immunostaining, analyzed by ImmuoRatio quantitative image software [9], showed a $20 \%$ proliferative index. The immunohistochemical findings were highly consistent with SFT. Based on the microscopic features including increased cellularity, cellular atypia, frequent mitoses, a high proliferative index, necrosis and absence of dedifferentiation, a diagnosis of de novo malignant solitary fibrous tumor was established.

\section{Discussion}

SFT is a relatively uncommon but distinctive mesenchymal neoplasm, originally described in the pleura cavity and later reported to occur ubiquitously [1]. Although

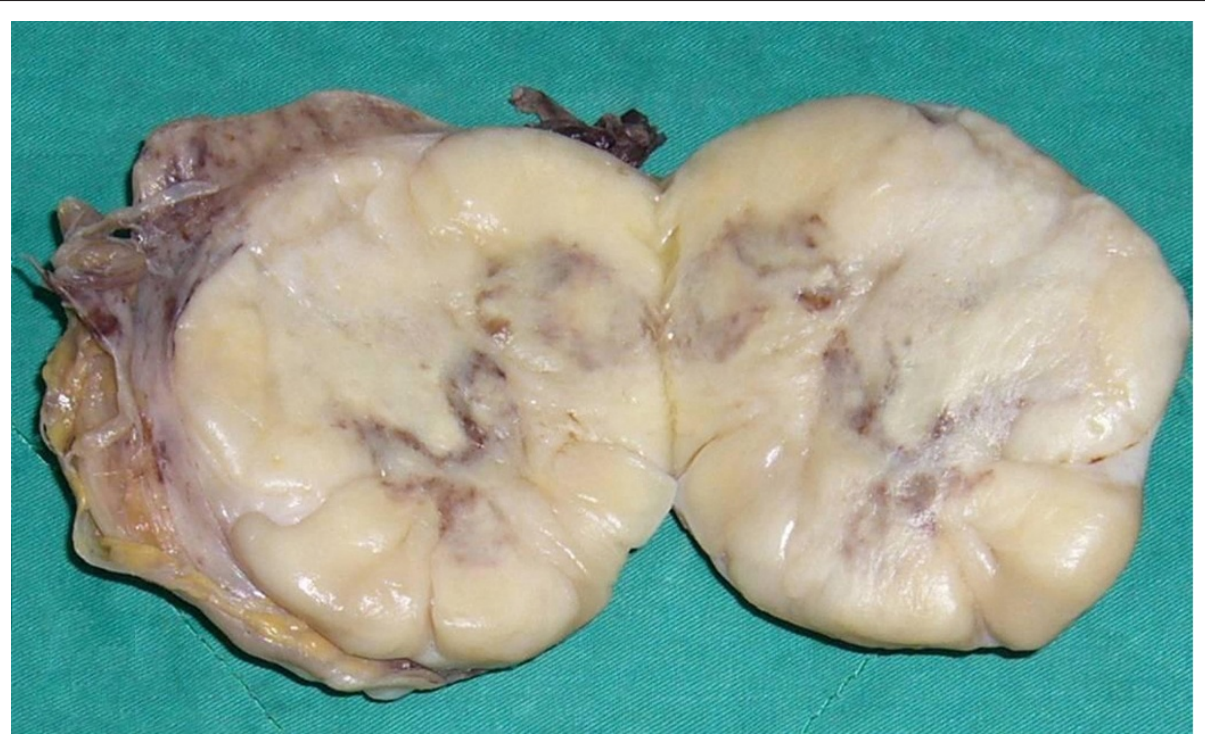

Figure 2 Gross morphology. The tumor was firm and showed a yellowish white to tan-gray, myxoid and lobulated cut surface with prominent hemorrhage and necrosis in the center. 

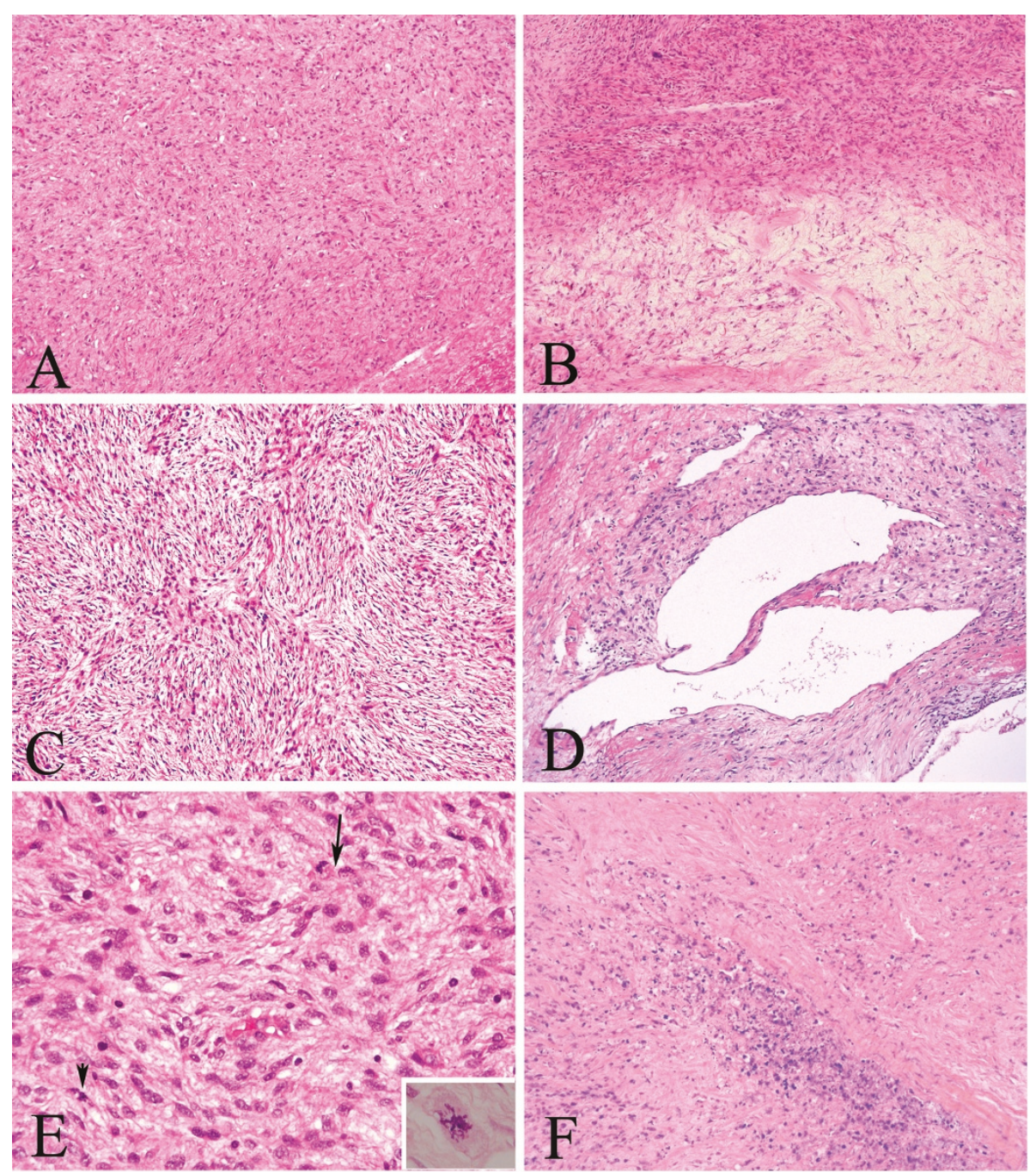

Figure 3 Photomicrographs. A, Proliferation of spindle cells arranging in a patternless architecture ( $\times 200$ original magnification). B, Alternating hypercellular and hypocellular areas of spindle cells separated from each other by bands of collagen fiber ( $\times 200$ original magnification). C, Spindle cells forming haphazard, storiform, or short fascicular arrangements in a loose myxoid to fibrous stroma containing dense collagen fibers (×200 original magnification). D, Hemangiopericytoma-like staghorn-like vessels (×200 original magnification). E, Tumor cells displaying mild to moderate atypia and 3 mitoses in this high power field (arrow and arrowhead) ( $\times 400$ original magnification). Abnormal mitoses were

occasionally seen (inset, $\times 400$ original magnification). F, Prominent tumor necrosis ( $\times 400$ original magnification).

the histogenesis of SFT remains undetermined, recent studies strongly favor a primitive mesenchymal or perivascular cell origin [10]. The kidney is a relatively infrequent site for SFT, with approximately at least 36 cases of renal SFT reported in a review article [7]. Clinically, these cases were frequently considered to be malignant due to their large tumor size by physical examinations and radiographic studies. Symptoms do not differ from those reported by patients with renal cell carcinoma. Hypoglycemia, which is a rare symptom in intrathoracic and extrathoracic SFTs, was not reported in any renal SFTs including our case [2].
Malignant behaviors in the form of recurrence and/or metastases can occur in $10 \%$ to $15 \%$ of intrathoracic SFTs and up to $10 \%$ of extrathoracic SFTs [2,3]. Malignant SFT is postulated to develop via two pathways: (1) de novo occurrence or (2) dedifferentiation or sarcomatous overgrowth from a pre-exsisting histologically benign SFT [1,4]. Most renal SFTs were classified as histologically benign and showed a favorable prognosis, with no evidence of recurrence during a follow-up period ranging from 2 to 89 months [7]. To our knowledge, two cases of malignant renal SFTs developing via dedifferentiation or sarcomatous overgrowth from a pre-existing 

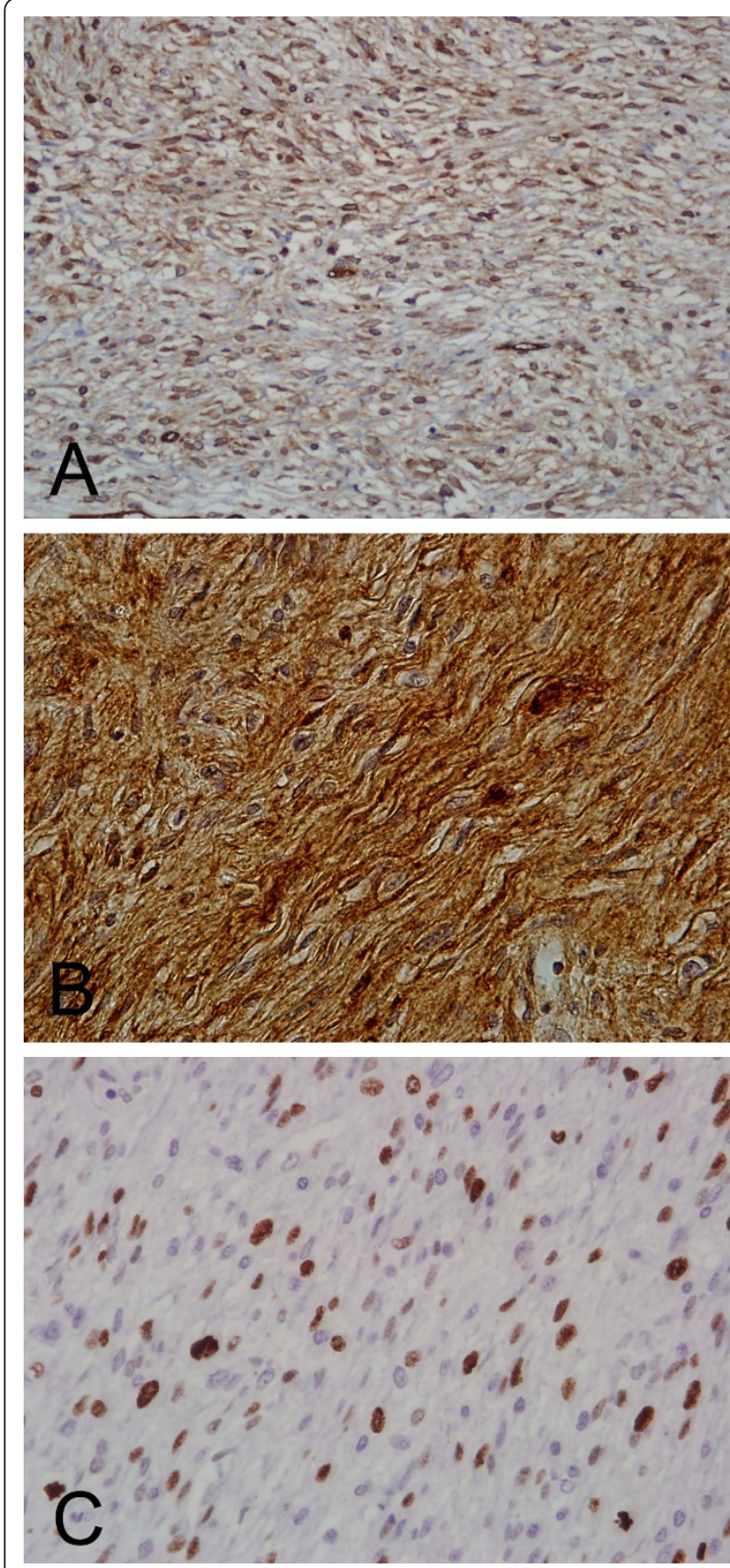

Figure 4 Immunohistochemical photomicrographs. Immunohistochemicaly, the tumor cells showing A, Weak to moderate CD34 immunostaining ( $\times 200$ original magnification). B, Strong and diffuse CD99 immunostaining ( $\times 200$ original magnification). C, Ki-67 immunostaining, analyzed by ImmuoRatio quantitative image software, showing a $20 \%$ proliferative index ( $\times 400$ original magnification).

benign SFT have been reported by Margo et al. and Fine et al., respecvievely $[7,8]$. A detailed comparison of the clinicopatholgoic features of these two cases and ours is summarized in Table 1. The tumor reported by Fine et al. had infiltrative borders and focal necrosis, and invaded beyond the renal capsule. The tumor described by Margo et al. was a 9-cm circumscribed mass devoid of either hemorrhage or necrosis. Notably, there was a 3-cm nodular area within the main tumor, microscopically responding to sarcomatous overgrowth. In contrast, our tumor showed a homogeneous cut surface with prominent necrosis and hemorrhage. Microscopically, the tumor reported by Fine et al. and Margo et al. showed typical features of benign SFT with $90 \%$ and 30\% of dedifferentiation or sarcomatous overgrowth, respectively. In contrast, our tumor appeared to develop de novo since we did not find any areas of dedifferentiation after extensive tumor sampling.

The criteria for clinical malignancy in intrathoracic SFT, first proposed by England et al. in 1989, include increased cellularity, pleomorphism, mitotic count more than 4 per 10 high power fields, necrosis, hemorrhage, size more than $10 \mathrm{~cm}$, non-pedunculated and atypical locations (parietal pleura, pulmonary parenchyma) [5]. The diagnostic criteria for malignant extrathoracic SFTs are purely microscopic and include increased cellularity, pleomorphism and mitotic count more than 4 per 10 high power fields $[4,11]$. Currently the impact of tumor characteristics (size, hemorrhage, necrosis and location) in predicting clinical malignancy in extrathoracic SFTs remains to be investigated. Our case fulfilled the diagnostic criteria for malignant extrathoracic SFTs. Additionally, the presence of hemorrhage and necrosis and a $20 \%$ Ki-67 proliferative index further supports a diagnosis of malignant renal SFT.

SFTs show a wide variety of microscopic growth patterns and should be distinguished from benign and malignant spindle cell tumors. Positive immunoreactivity for CD34 and CD99 is characteristic of SFT, and highly valuable in differentiating from other mesenchymal tumors $[2,4]$. However the expression of CD34 and CD99 may be decreased or absent in areas with marked atypia or dedifferentiation $[8,12]$. Genetic analyses of SFT to date have not found consistent and characteristic cytogenetic abnormalities that can be used an ancillary diagnostic marker. Missense mutation of platelet-derived growth factor receptor- $\beta$ (PDGFR- $\beta$ ) has been reported in 2 of 88 pleuro-pulmonary SFTs [13], but we did not detect PDGFR- $\beta$ mutation in our case (data not shown).

The prognosis of extrapleural SFTs is more unpredictable than that of pleural tumors due to lack of largescale studies. The clinical outcomes were rather strikingly different in the two malignant renal SFTs with dedifferentiation. While a patient developed multiple small lung nodules 4 months post-operatively [8], the other patient was disease-free after 15 months of followup [7]. Our patient has been well without evidence of recurrence or metastasis for 30 months. Some studies 
Table 1 Comparsion of the clinicopathologic features of malignant renal solitary fibrous tumor

\begin{tabular}{llll}
\hline & Fine et al. & Margo et al. & Present case \\
\hline Age $(\mathrm{yr})$ & 76 & 34 & 50 \\
\hline Gender & Female & Female & Female \\
\hline Location & Left kidney & Left kidney & Right kidney \\
\hline Size & $12 \times 10 \times 7.5 \mathrm{~cm}$ & $9 \mathrm{~cm}$, with a distinct 3-cm nodule & $9 \times 9 \times 6 \mathrm{~cm}$ \\
\hline Tumor borders & Infiltrative with invasion beyond the renal capsule & Circumscribed & Circumscribed, unencapsulated \\
\hline Necrosis & Focal & Absent & Marked \\
\hline Hemorrhage & NA & Absent & Present \\
\hline Histology & $10 \%$ benign SFT 90\% dedifferentiation & $70 \%$ benign SFT 30\% dedifferentiation & De novo malignant SFT \\
\hline Mitoses & Frequent & $2-6 / 10$ HPF & $8 / 10$ HPF \\
\hline Atypia & Marked in areas of dedifferentiation & Marked in areas of dedifferentiation & Mild to moderate \\
\hline Ki-67 proliferative index & NA & $1-4 \%$ & $20 \%$ \\
\hline CD34 & Loss of expression in areas of dedifferentiation & Positive & Positive \\
\hline Follow-up & Multiple lung nodules 4 month after nephrectomy & 15 months, NED & 30 months, NED \\
\hline
\end{tabular}

NA: not available, NED: no evidence of disease

indicated that extrapleural SFTs had similar prognosis as the pleural counterpart, and a tumor with malignant histological feature was associated with recurrence and metastasis $[3,14]$, but other studies showed that extrapleural SFTs tended to have a favorable outcome than pleural ones, even those with malignant histological features $[11,15]$. Studies also showed that deep-seated locations, over-expression of p53 and p16, loss of CD34 immunostaining and the presence of dedifferentiated areas may indicate more aggressive behaviors [6]. Complete surgical excision and long-term follow-up are generally recommended for patients with extrapleural SFTs [15]. Besides, due to the rich vascularity and possible origin from pericytes, antiangiogenic therapy, especially for the advanced cases is also under clinical investigation [16].

\section{Consent}

Written informed consent was obtained from the patient for publication of this case report and accompanying images. Submission of this case report was approved by Institutional Review Board (IRB) of Chang Gung Memorial Hospital, Tao-Yuan, Taiwan. A copy of the written consent is available for review by the Editor-in-Chief of this journal.

\section{List of abbreviations}

SFT: solitary fibrous tumor

\section{Acknowledgements}

The authors appreciate Drs. Jonathan I. Epstein and Elizabeth Montgomery at the Department of Pathology, Johns Hopkins Medical Institutions, Baltimore, MD, USA for confirming our diagnosis.

\section{Author details}

${ }^{1}$ Department of Pathology, Chang Gung Memorial Hospital, Keelung, Taiwan. ${ }^{2}$ Department of Urology, Chang Gung Memorial Hospital, Keelung, Taiwan.
${ }^{3}$ Department of Radiology, Chang Gung Memorial Hospital, Keelung, Taiwan. ${ }^{4}$ College of Medicine, Chang Gung University, Tao-yuan, Taiwan.

\section{Authors' contributions}

TYH was responsible for data collection, literature search and manuscript preparation. YCCC carried out gross and microscopic examinations. WHC performed the surgery and clinical follow-up of the patient. CSC interpreted pre-operative and follow-up imaging studies. CLC, CCH and HPC participated in the microscopic analyses and helped making a final diagnosis. JRC participated in manuscript preparation and approved the final manuscript. All authors read and approved the final manuscript.

\section{Competing interests}

The authors declare that they have no competing interests.

Received: 10 August 2011 Accepted: 5 October 2011

Published: 5 October 2011

\section{References}

1. Chan JK: Solitary fibrous tumour-everywhere, and a diagnosis in vogue Histopathology 1997, 31:568-76.

2. Weiss SW, Goldblum JR: Soft tissue tumors of intermediate malignancy of uncertain type. In Soft Tissue Tumor.. 5 edition. Edited by: Weiss SW, Goldblum JR. Philadephia: Mosby Elsevier; 2008:1093-1160.

3. Vallat-Decouvelaere AV, Dry SM, Fletcher CD: Atypical and malignant solitary fibrous tumors in extrathoracic locations: evidence of their comparability to intra-thoracic tumors. Am J Surg Pathol 1998, 22:1501-11.

4. Guillou LFJ, Fletcher CDM, Mandahi N: Extrapleural solitary fibrous tumour and hemangiopericytoma. In World Health Organization Classification of Tumours: Pathology and Genetics of Tumours of Soft Tissue and Bone. Edited by: Fletcher CDM, Unni KK, Mertens F. Lyon: IARCPress; 2002:86-90.

5. England DM, Hochholzer L, McCarthy MJ: Localized benign and malignant fibrous tumors of the pleura. A clinicopathologic review of 223 cases. Am J Surg Pathol 1989, 13:640-58.

6. Mosquera JM, Fletcher CD: Expanding the spectrum of malignant progression in solitary fibrous tumors: a study of 8 cases with a discrete anaplastic component-is this dedifferentiated SFT? Am J Surg Pathol 2009, 33:1314-21.

7. Magro G, Emmanuele C, Lopes M, Vallone G, Greco P: Solitary fibrous tumour of the kidney with sarcomatous overgrowth. Case report and review of the literature. APMIS 2008, 116:1020-5.

8. Fine SW, McCarthy DM, Chan TY, Epstein JI, Argani P: Malignant solitary fibrous tumor of the kidney: report of a case and comprehensive review of the literature. Arch Pathol Lab Med 2006, 130:857-61.

9. Tuominen VJ, Ruotoistenmaki S, Viitanen A, Jumppanen M, Isola J: ImmunoRatio: a publicly available web application for quantitative 
image analysis of estrogen receptor (ER), progesterone receptor (PR), and Ki-67. Breast Cancer Res 2010, 12:R56.

10. Ide F, Obara K, Mishima K, Saito I, Kusama K: Ultrastructural spectrum of solitary fibrous tumor: a unique perivascular tumor with alternative lines of differentiation. Virchows Arch 2005, 446:646-52.

11. Hasegawa T, Matsuno Y, Shimoda T, Hasegawa F, Sano T, Hirohashi S: Extrathoracic solitary fibrous tumors: their histological variability and potentially aggressive behavior. Hum Pathol 1999, 30:1464-73.

12. Yokoi T, Tsuzuki T, Yatabe Y, Suzuki M, Kurumaya H, Koshikawa T, Kuhara H, Kuroda M, Nakamura N, Nakatani Y, et al: Solitary fibrous tumour: significance of p53 and CD34 immunoreactivity in its malignant transformation. Histopathology 1998, 32:423-32.

13. Schirosi L, Lantuejoul S, Cavazza A, Murer B, Yves Brichon P, Migaldi M, Sartori G, Sgambato A, Rossi G: Pleuro-pulmonary solitary fibrous tumors: a clinicopathologic, immunohistochemical, and molecular study of 88 cases confirming the prognostic value of de Perrot staging system and p53 expression, and evaluating the role of c-kit, BRAF, PDGFRs (alpha/ beta), c-met, and EGFR. Am J Surg Pathol 2008, 32:1627-42.

14. Brunnemann RB, Ro JY, Ordonez NG, Mooney J, El-Naggar AK, Ayala AG: Extrapleural solitary fibrous tumor: a clinicopathologic study of 24 cases. Mod Pathol 1999, 12:1034-42.

15. Morimitsu Y, Nakajima M, Hisaoka M, Hashimoto H: Extrapleural solitary fibrous tumor: clinicopathologic study of 17 cases and molecular analysis of the p53 pathway. APMIS 2000, 108:617-25.

16. Park MS, Araujo DM: New insights into the hemangiopericytoma/solitary fibrous tumor spectrum of tumors. Curr Opin Oncol 2009, 21:327-31.

doi:10.1186/1746-1596-6-96

Cite this article as: Hsieh et al:: De novo malignant solitary fibrous tumor of the kidney. Diagnostic Pathology 2011 6:96.

\section{Submit your next manuscript to BioMed Central} and take full advantage of:

- Convenient online submission

- Thorough peer review

- No space constraints or color figure charges

- Immediate publication on acceptance

- Inclusion in PubMed, CAS, Scopus and Google Scholar

- Research which is freely available for redistribution

Submit your manuscript at www.biomedcentral.com/submit
Ciomed Central 